\title{
Detección de anomalías mediante espectrometría de imagen en la coordillera volcánica central de Costa Rica. Implicaciones en la exploración planetaria
}

Juan Gregorio Rejas Ayuga, Rubén Martínez Marín, Javier Bonatti, Jesús Martínez-Frías y Miguel Marchamalo Sacristán

\section{Resumen}

El objetivo del presente trabajo es el estudio comparativo de métodos de cálculo de anomalías espectrales a partir de espectrometría de imagen en distintas áreas de test de la Cordillera Volcánica Central (CVC) de Costa Rica. En la detección de respuestas anómalas se asume un no conocimiento previo de los objetivos, de tal manera que los píxeles se separan automáticamente en función de su información espectral significativamente diferenciada respecto de un fondo que se estima, bien de manera global para toda la escena, bien localmente por segmentación de la imagen.

Se han utilizado rangos del espectro visible-infrarrojo cercano (VNIR), infrarrojo de onda corta (SWIR) y térmico (TIR) de cubos de datos hiperepectrales procedentes de los sensores aeroportados HyMAP y MASTER. Se han diseñado experimentos sobre escenarios naturales de la CVC, urbanos y semiurbanos de diferente complejidad, analizando el comportamiento del detector de anomalías estándar RX y métodos distintos basados en proyección de imagen y reducción de la dimensionalidad. Se discuten los resultados obtenidos y se realiza una aproximación sobre la implicación como homólogos terrestres en exploración planetaria.

Palabras clave: Anomalías espectrales. Hiperespectral. Reconocimiento automático de patrones. Exploración planetaria.

\section{Abstract}

The aim of this work is the comparative study of methods for calculating spectral anomalies from imaging spectrometry test in several areas of the Central Volcanic Range (CVC) of Costa Rica. In the detection of anomalous responses is assumed 
no prior knowledge of the targets, so that the pixels are automatically separated according to their spectral information significantly differentiated with respect to a background to be estimated, either globally for the entire scene, either locally by the image segmentation.

We used spectral ranges of visible-near infrared (VNIR), shortwave infrared (SWIR) and thermal (TIR) data cubes hiperepectrales from HyMAP airborne sensors and MASTER. We have designed experiments on natural scenes of the CVC, urban and semi-urban of different complexity, analyzing the behavior of the standard RX anomaly detector and different approaches based on imaging and dimensionality reduction. We discuss the results and is an approximation of the involvement as terrestrial counterparts in planetary exploration.

Keywords: Spectral Anomaly. Hydrothermal Alteration. Hyperspectral. Multisensor.

Juan Gregorio Rejas Ayuga (rejasaj@inta.es, juangregorio.rejas@upm.es), Instituto Nacional de Técnica Aeroespacial, INTA; Universidad Politécnica de Madrid, UPM. Rubén Martínez Marín (ruben.martinez@upm.es), Universidad Politécnica de Madrid, UPM. Javier Bonatti (jbonatti2011@gmail.com), Universidad de Costa Rica, UCR. Jesús Martínez-Frías (jmfrias@cab.inta-csic.es), Centro de Astrobiología CAB, INTA-CSIC; Universidad Politécnica de Madrid, UPM. Miguel Marchamalo Sacristán (miguel.marchamalo@ upm.es), Universidad Politécnica de Madrid, UPM. 


\section{INTRODUCCIÓN}

La detección de anomalías espectrales tiene por objeto extraer de manera automática píxeles de imagen que responden significativamente diferente respecto su entorno. Distintos métodos (ver Bibliografía) se han desarrollado en las últimas décadas que han supuesto la mejora en el establecimiento de relaciones entre la dimensionalidad de los datos hiperespectrales y la optimización de los procesos de búsqueda (Duran y Petrou, 2007; Shaum, 2005; Stein et al., 2002), así como la diferenciación sub píxel de la mezcla espectral y su implicación en las respuestas anómalas (Bar et al., 2010; Eismann et al., 2009; Manolakis y Shaw, 2002).

En otro sentido, la espectrometría de imagen se ha mostrado eficaz para la caracterización de minerales en base a métodos estadísticos que utilizan las bandas reflectivas y absortivas diagnóstico de estos. Configuraciones hiperespectrales en rangos del infrarrojo de onda corta (SWIR), infrarrojo cercano y visible (VNIR) han permitido cartografiar materiales de alteración hidrotermal en distintos escenarios geológicos (Antón-Pacheco et al., 2001; Crosta et al., 2003). Trabajos previos han demostrado la aplicación en mineralogía de rangos del espectro electromagnético en el infrarrojo térmico y fusión en la región de las microondas para detectar contaminación, materiales enterrados (Nasrabadi, 2008; Rejas et al., 2010), etc.

Un aspecto relevante en nuestro estudio es establecer relaciones que permitan conectar las anomalías espectrales con lo que podemos denominar anomalías informacionales. Es decir, información relacionada con la respuesta anómala más allá de ser producto de una diferenciación significativa respecto de un fondo. Interesa por lo tanto conocer qué información nos proporciona la respuesta anómala detectada y caracterizar en qué condiciones se produce.

Se presenta en este sentido una aproximación multifuente, como estudio de la correlación entre anomalías espectrales y materiales de alteración hidrotermal en los complejos volcánicos de Turrialba, Irazú y Poás, situados en la Cordillera Volcánica Central (CVC) de Costa Rica. Asimismo se analiza la influencia de la resolución espectral en la determinación del fondo, aspecto crítico en el cálculo de anomalías. Se comparan los resultados con índices de vegetación y suelo en una zona con actividad geotérmica y vulcanológica creciente, por lo que, debido a la cercanía de núcleos urbanos, hacen especialmente necesario un seguimiento en la evolución de su estado.

Finalmente, se avanza el estudio de bandas diagnóstico de espectros y su relación como valores atípicos en la detección de anomalías para un análisis 
espectral automatizado en geología y exploración planetarias.

\section{DATOS Y PRE PROCESO}

Se han analizado datos de sensores aeroportados y satelitales en una estrategia multiescala. Se ha utilizado imágenes de reflectancia del sensor HyMAP (Cocks et al., 1998) y del sensor MASTER (Hook et al., 2001), adquiridas ambas el 7 de marzo de 2005 sobre el volcán Turrialba y entorno. La escena HyMAP (125 bandas entre $0.4589 \mathrm{~mm}$ y $2.491 \mathrm{~mm}$ ) tiene una dimensión de 710 × 2415 píxeles, con una resolución espacial de $15 \mathrm{~m}$. La escena MASTER es de 1650 x 4466 píxeles, de resolución espacial de $9 \mathrm{~m}$ y codificación radiométrica 16 bit. Los 50 canales de imagen de MASTER se agrupan en un puerto de 25 canales en el VNIRSWIR $(0.463 \mathrm{~mm}$ a $2.427 \mathrm{~mm})$, y 25 canales entre $3.075 \mathrm{~mm}$ y $13 \mathrm{~mm}$ del MIR-TIR.

- Se han utilizado escenas ASTER comprendidas en un período entre 2002 y 2010, y una escena Hyperion del 5 de marzo de 2010 adquiridas a través de EarthExplorer del USGS (http://earthexplorer. usgs.gov/).

- Se ha aplicado un algoritmo MNF (Minimum Noise Fraction) que ha permitido reducir el ruido en las imágenes HyMAP y MASTER. Se han desechado los canales de los sensores que presentaban una relación señal/ruido elevada, canales 62 a 65 y 125 en el caso de HyMAP, y canales 16 a 19, 25 a 41 y 50 para las imágenes MASTER.

Las imágenes aeroportadas han sido georreferenciadas directamente mediante el algoritmo GLT de ENVI, utilizando para ello la geometría calculada a partir de datos de posición y orientación medidos por sistemas inerciales GPS/IMU en el momento de adquisición sobre el área de estudio.

\section{ANÁLISIS EXPLORATORIOS}

Se han aplicado técnicas exploratorias de imagen cuyo objetivo es contrastar parámetros biofísicos y obtener una primera aproximación del estado de la vegetación y los suelos en el entorno de Turrialba.

Se ha realizado un chequeo de las transformaciones radiométricas y geométricas de las imágenes a partir de datos medidos en campañas de supervisión. En agosto de 2010 y febrero de 2012 se ha llevado a cabo un reconocimiento sobre el terreno, que ha permitido muestrear 29 y 20 puntos respectivamente, medidos en campo y en laboratorio con radiómetros USB400 y ASD FieldSpec 
Hi-Re. Los espectros se han utilizado para caracterizar los materiales de alteración hidrotermal y para comprobar la reflectancia de las imágenes HyMAP y MASTER mediante una regresión empírica lineal.

Se ha contrastado la georreferenciación de las imágenes mediante 50 check points medidos en el terreno en proyección cartográfica UTM zona 17 y Datum WGS84, obteniendo un error absoluto planimétrico de 3.2 y 1.9 píxeles, respectivamente para las imágenes MASTER y HyMAP.

Se han calculado índices de vegetación y de suelo para el conjunto de las imágenes. Se han calculado tres índices de vegetación a partir de los valores de reflectancia de las imágenes hiperespectrales. Se ha generado el NDVI (Rouse et al., 1974) para evaluar la estimación de la cubierta (IAF), y el cociente (Berni et al., 2010) entre el índice de reflectancia transformada por la absorción de la clorofila (TCARI) y el índice de vegetación del suelo ajustado optimizado (OSAVI).

$$
N D V I=\frac{\rho_{\mu N I R}-\rho_{\mu R}}{\rho_{\mu N I R}-\rho_{\mu R}}
$$

Donde $P_{\mu N \mathbb{R}}=$ reflectancia de banda del infrarrojo próximo

$$
\begin{aligned}
& \text { TCARI / OSAVI } \\
& =3 \cdot\left[\left(\rho_{\mu 700}-\rho_{\mu 670}\right)-0.2 \cdot\left(\rho_{\mu 700}-\rho_{\mu 550}\right) \cdot\left(\rho_{\mu 700}-\rho_{\mu 670}\right)\right] \\
& (1+0.16) \cdot\left(\rho_{\mu 800}-\rho_{\mu 670}\right) /\left(\rho_{\mu 800}-\rho_{\mu 670}+0.16\right)
\end{aligned}
$$

Donde $\rho_{\mu \bar{s}}=$ reflectancia de la banda i.

Con ello se pretende evaluar la influencia de la cubierta de vegetación, abundante en la zona de estudio, en el análisis de imagen posterior, y estimar el impacto de la actividad de los complejos volcánicos en las laderas y zonas próximas. Se observa en los índices calculados a partir de la serie temporal de escenas ASTER, el efecto acusado de lluvia ácida sobre el vigor en la vegetación en una marcada dirección oeste al noroeste de los cráteres.

\section{ANÁLISIS HIPERESPECTRAL Y DETECCIÓN DE ANOMALÍAS}

\subsection{Alteración hidrotermal en la CVC}


Existen minerales claramente indicativos de la presencia de alteración hidrotermal que podemos detectar remotamente a partir de su respuesta espectral en bandas diagnóstico de absorción y reflexión (Antón-Pacheco et al., 2001; Bataller et al., 2010). Estas características espectrales que los hacen únicos, las podemos extraer de imágenes hiperespectrales convenientemente calibradas. Minerales arcillosos, tales como caolinita, illita y alunita, presentan una alta reflectancia entre las longitudes de onda de $1,55 \mathrm{~mm}$ y $1,75 \mathrm{~mm}$, y una alta absorción entre de $2,08 \mathrm{~mm}$ y $2,35 \mathrm{~mm}$. Otra característica adicional para identificar estos minerales es que las rocas que no han sufrido el proceso hidrotérmico, por lo general presentan valores normales en las longitudes de onda antes mencionadas. Los minerales con alto contenido en Fe presentan un contraste muy elevado entre las longitudes de onda $0,63 \mathrm{~mm}$ y $0,69 \mathrm{~mm}$ y longitudes de onda de $0,45 \mathrm{~mm}$ a las $0,52 \mathrm{~mm}$.

Hemos seleccionado cuatro variables de imagen para analizar sus componentes principales (ACP) diferenciados por grupos de bandas, dos altamente reflectantes y dos altamente absorbentes para cada mineral (Crosta et al., 2004). En el caso de las imágenes HyMAP se han seleccionado las siguientes bandas en los sucesivos PCA: para illita, canales 6, 25, 105 y 108; para alunita canales 6, 25, 105 y 116; para caolinita canales 6, 81, 108 y 116; para caolinita + esmecita canales $6,81,108,119$. Para el grupo de minerales de Fe, como la goethita, hematita y jarosita, se han utilizado los canales HyMAP 4, 32, 62 y 103 y para la montmorillonita los canales $4,25,81$ y 94 .

Figura 1: Abundancia de minerales de alteración hidrotermal (Alu-cyan, Illi-amarillo, Caol-morado, Caol+Esme-morado 2, Mont-marrón) a partir de HyMAP, detalle en el volcán Turrialba de la CVC (a) y MASTER, de talle de la ciudad de Turrialba (b)

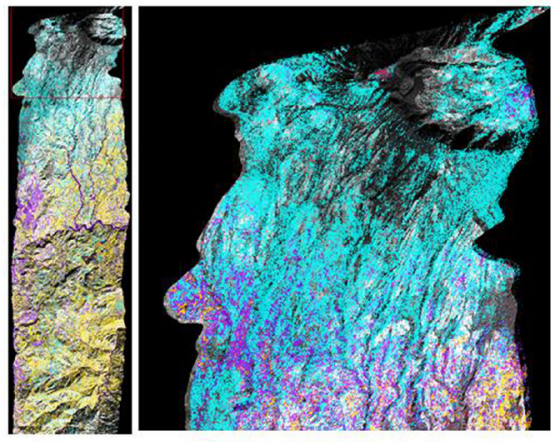

(a)
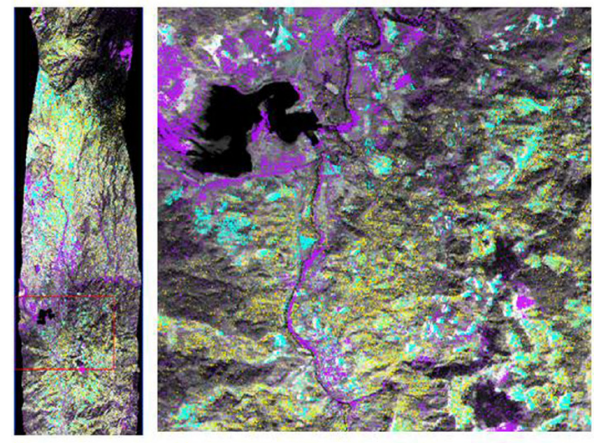

(b)

En el caso de de las imágenes MASTER se han realizado análisis de componentes principales diferenciados para cada mineral a partir de las siguientes lon- 
gitudes de onda: canales 4, 8, 21 y 24 para alunita; canales 4, 8, 21 y 22 para illita; canales 4, 1322 y 24 para caolinita.

Los resultados obtenidos para HyMAP y MASTER se han clasificado mediante el algoritmo K-Means, calculando matrices de confusión a partir del muestreo de campo como valor real. Para HyMAP se ha obtenido un solape de precisión al $82.56 \%$ y coeficiente Kappa de 0.75 . Para MASTER se ha obtenido un solape de precisión al $75.11 \%$ y coeficiente Kappa de 0.69 .

Los resultados están muy influenciados por la cubierta vegetal, que actúa como aporte erróneo de reflectancia en el cálculo de las nuevas variables o CPs. Sin embargo, se observa la escasa presencia de materiales arcillosos de alteración fílica, lo que contrasta con la abundancia de los componentes de óxido de hierro.

\subsection{Detección de anomalías espectrales en la CVC}

Se ha aplicado al conjunto de las imágenes el algoritmo RX (Reed y Xaoli, 1996) admitido ampliamente como un estándar en la detección de anomalías espectrales. Se han contrastado las anomalías obtenidas por RX con las calculadas mediante projection pursuit (Malpica et al., 2008), mediante un método SSRX basado en subespacios hiperespectrales y mediante ortoproyección subespacial - OSPRX (Borghys et al. 2012). Para todos los métodos se ha separado el cálculo por rangos de canales reflectivos y emisivos. En el caso de las imágenes MASTER, se ha focalizado en los canales térmicos de MASTER fusionados con fotografía aérea a $2.5 \mathrm{~m}$ de resolución, para mejorar la separabilidad de clases manteniendo la información espectral (Rejas et al., 2007). Se han obtenido 8 nuevas variables a partir de los canales MASTER 42 a 49. La variable 6 proyectada registra menor concentración de humedad, y se observa que corresponde a una región amplia y bien delimitada en el noroeste de Turrialba, para esta área analizada.

Se ha llevado a cabo además un reconocimiento de patrones. Para las imágenes MASTER y ASTER se ha calculado un índice térmico (Rejas et al., 2012) aprovechando la capacidad de separación entre las cubiertas del espectro emisivo de estos dos sensores. Se han generado convoluciones de imagen utilizando un filtro de mediana, que posteriormente han intervenido en un cociente entre las longitudes de onda $10.16 \mathrm{~mm}$ y $12.21 \mathrm{~mm}$, corregido por la razón entre las ganancias respectivas de los canales. La variable resultante se ha umbralizado para resaltar píxeles detectados como posibles anomalías. 
$T H I=\frac{\left\lfloor\operatorname{conv}\left(L_{\mu 10.16}\right)-\operatorname{conv}\left(L_{\mu 12.21}\right)\right\rfloor}{0.24 \cdot\left[\operatorname{con} v\left(L_{\mu 10.16}\right)-\operatorname{con} v\left(L_{\mu 1221}\right)\right]}$

Donde conv = imagen convolucionada y $L_{\mu}=$ radiancia en el sensor por longitud de onda.

Figura 2: Índice térmico THI (rojo-violeta) entre I $10.16 \mathrm{~mm}$ y $12.21 \mathrm{~mm}$ sobre canal 6 en nivel de gris de MASTER. Detalle Volcán Turrialba y laderas

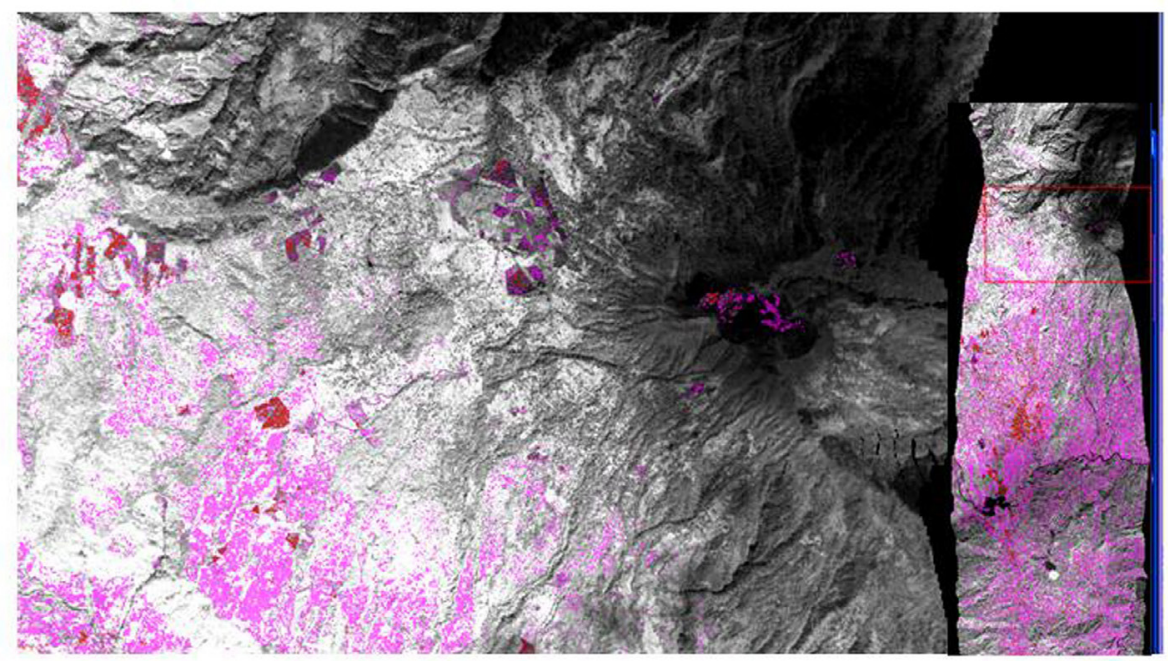

\subsection{Resultados y discusión}

Se ha estudiado la relación entre las anomalías espectrales y las alteraciones hidrotermales obtenidas en los anteriores apartados. Para ello se ha ajustado linealmente un espacio muestral de 35 pares de puntos, anomalías espectrales en el eje $Y$ y minerales alterados en el eje $X$. Todos las regresiones se han calculado a un nivel de confianza del $95 \%$, eliminando en cada ajuste los valores muestrales que presentaban residuos inusuales, que se correspondían principalmente con nubes y sombras. Los resultados obtenidos se resumen en las Tablas 1 y 2. 
Tabla 1: Comparación de relaciones entre anomalías espectrales y alteración hidrotermal a partir de imágenes HyMAP.

\begin{tabular}{|l|c|c|c|}
\hline \multicolumn{1}{|c|}{ Modelos para HyMAP } & P-valor & Correlación & $R^{2}$ \\
\hline $\mathrm{RX}=1101,61+9,86744^{*}$ Alunita & 0,0001 & 0,850365 & $72,31 \%$ \\
\hline $\mathrm{RX}=-1528,05-1,92404 *$ Illita & 0,0379 & $-0,554625$ & $30,76 \%$ \\
\hline $\mathrm{RX}=-611,046+1,03826^{*}$ Caolinita & 0,0515 & 0,511091 & $26,12 \%$ \\
\hline $\mathrm{RX}=739,318-7,59725^{*}$ Caol+Esmecita & 0,0182 & $-0,599314$ & $35,92 \%$ \\
\hline $\mathrm{RX}=834,65+3,50578^{*}$ minerales Fe & 0,0153 & 0,611924 & $37,45 \%$ \\
\hline $\mathrm{RX}=-796,88-1,47957^{*}$ Montmorollonita & 0,0851 & $-0,45922$ & $21,09 \%$ \\
\hline
\end{tabular}

Tabla 2: Comparación de relaciones entre anomalías espectrales y alteración hidrotermal a partir de imágenes MASTER

\begin{tabular}{|l|l|l|l|}
\hline Modelos para MASTER & P-valor & Correlación & $R^{2}$ \\
\hline $\mathrm{RX}=79,4678-0,579643^{*}$ Alunita & 0,0004 & $-0,811637$ & $65,88 \%$ \\
\hline $\mathrm{RX}=69,1488-0,50982 *$ Illita & 0,0001 & $-0,855083$ & $73,12 \%$ \\
\hline $\mathrm{RX}=126,893-0,905153^{*}$ Caolinita & 0,0178 & $-0,620723$ & $38,53 \%$ \\
\hline
\end{tabular}

De los modelos lineales generados, el que obtiene un más alto valor de R cuadrado de $73.12 \%$ se corresponde con el de RX- Illita para las imágenes de MASTER.

El valor $\mathrm{P}$ obtenido inferior a 0.05 en todos los casos, salvo para RX-caolinita y RX-montmorollonita a partir de HyMAP, indica que hay una relación estadísticamente significativa y no hay ninguna indicación de autocorrelación serial en los residuos en el nivel de confianza del $95.0 \%$.

El estadístico R-cuadrado indica que los modelos ajustados explican en diferentes porcentajes la variabilidad entre alteración hidrotermal y anomalías espectrales. Los coeficientes de correlación máximos (-0.831632 y 0.850365) indican una relación moderadamente fuerte entre las correspondientes variables. 
Figura 3: Detalle anomalías espectrales SSRX (a), Alunita (b) e Illita (c) de HyMAP 2005 (Superior). Detalle de anomalías espectrales OSPRX (a), Alunita (b) e Illita (c) de MASTER
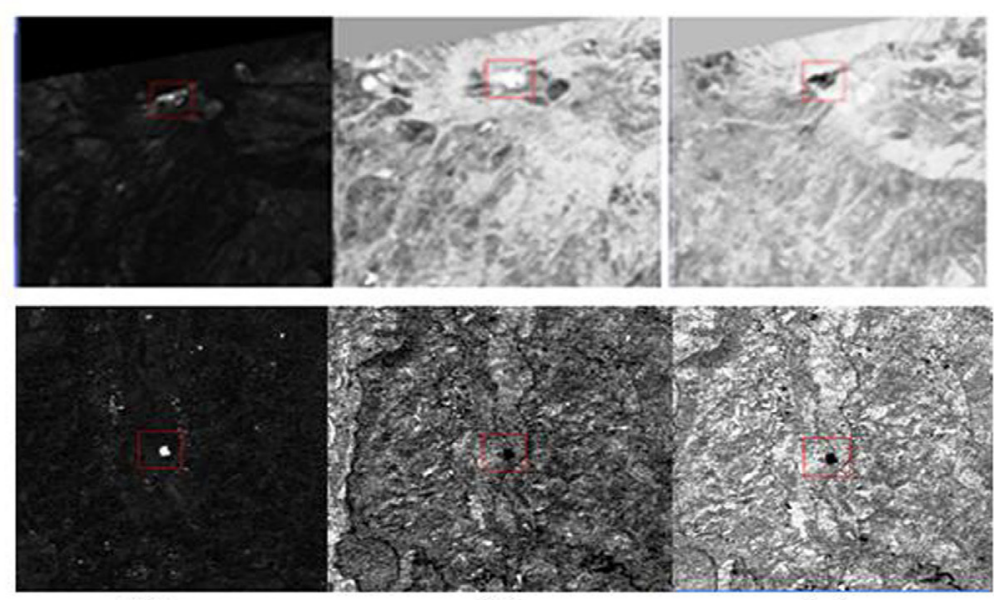

(a)

(b)

(c)

Existe correlación, positiva o negativa dependiendo del caso, entre las alteraciones hidrotermales detectadas y las anomalías espectrales calculadas a partir de los canales reflectivos de HyMAP.

En el caso de las anomalías térmicas, no es tan consistente esta relación. Sin embargo, esta sí se evidencia con parámetros biofísicos calculados y con efectos asociadas a la actividad de los volcanes. Un caso particular es el del propio cráter, que se detectan como anomalías térmicas, pero pasan casi desapercibidos como anomalías espectrales en el rango reflectivo.

La alteración hidrotermal detectada a partir de HyMAP en CVC y entorno, está vinculada a áreas de cultivos o suelo desnudo y a superficies artificiales (cubiertas de edificaciones principalmente). Esto no implica que en los alrededores de estas zonas no existan minerales de alteración hidrotermal. Se observa que la detección está fuertemente influenciada por el aporte de la amplia, densa y homogénea cobertura natural de vegetación en la información espectral registrada por los sensores espaciales. 
Figura 4: Anomalías RX de canales VNIR (izqda.) y TIR de MASTER (centro). Comparación de métodos de detección de anomalías RX para MASTER: (1) Todos los canales, (2) sólo VNIR, (3)
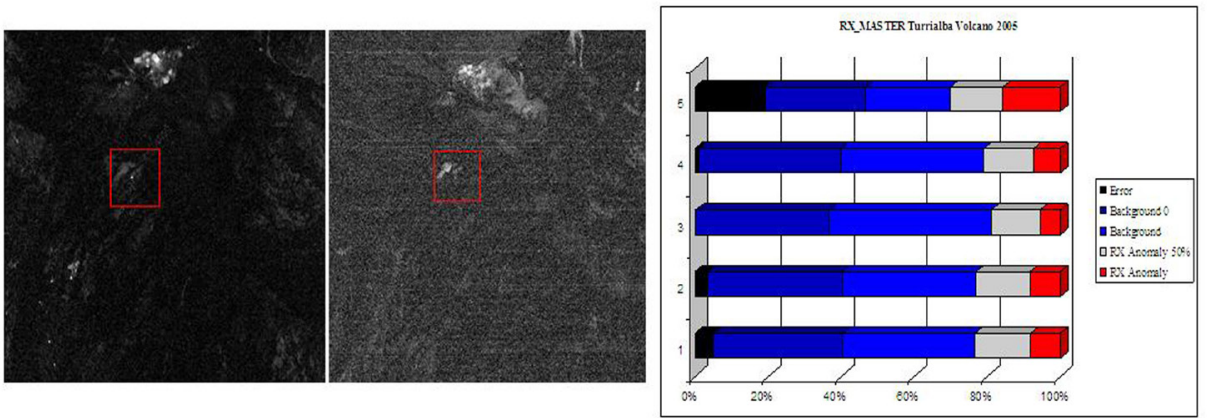

Se ha analizado la influencia de las bandas de absorción y reflexión en la detección de anomalías espectrales por los diferentes métodos mencionados. La diferencia entre las anomalías espectrales con y sin bandas de diagnóstico en el VNIR para el caso HyMAP es el 0,75\%, y para el caso de MASTER es el 0,84\%, mientras que la diferencia para la detección del fondo espectral es de $-2,52 \%$ y $-2,32 \%$, respectivamente.

\section{CONCLUSIONES}

Se ha realizado un estudio comparativo de distintos métodos de detección de anomalías espectrales a partir de imágenes HyMAP y MASTER en áreas de test de la Cordillera Volcánica Central (CVC) de Costa Rica.

Se ha comprobado que la mayor resolución espectral de las imágenes mejora la precisión en la detección de bandas diagnóstico de los minerales de alteración hidrotermal analizados. Una mayor dimensionalidad de los datos implica no obstante un mayor número de muestras para caracterizar clases de materiales.

Mayores concentraciones de minerales de alteración hidrotermal, en escenarios en los que las fuentes de error (principalmente la vegetación) se minimizan, están correlacionadas con las anomalías calculadas en el rango espectral reflectivo. No se ha establecido una relación clara entre anomalías térmicas y minerales analizados.

La mezcla espectral, asociada directamente con la resolución espacial de los sensores aeroportados y satelitales utilizados, repercute significativamente en 
la caracterización del fondo, y consiguientemente en las anomalías espectrales calculadas. Los resultados obtenidos por los distintos métodos de detección de anomalías espectrales desarrollados se ven afectados así mismo por la selección de bandas de absorción y reflexión, mejorando estos al considerar en el cálculo la respuesta en longitudes de onda diagnóstico de determinados materiales.

Finalmente, los resultados obtenidos permiten considerar adecuadas determinadas áreas de la CVC como análogos terrestres en exploración planetaria mediante teledetección hiperespectral.

\section{BIBLIOGRAFÍA}

- Antón-Pacheco, C., Rowan, L.C., Mars, J.C. and Gumiel, J.C., (2001). "Characterization of mine materials and hydrothermally altered rocks in the rio Tinto minning districy (southwest Spain) using HyMAP data". Revista de Teledetección. Number 16: 65-68 pp.

- Bar, D.O., Wolowelsky, K., Swirski, Y., Figov, Z., Michaeli, A., Vaynzof, Y., Abramovitz, Y., Ben-Dov, A., Yaron, O., Weizman, L. and Adar, R. (2010). "Target detection and verification via airborne hyperspectral and high-resolution imagery processing and fusion". leee Sensors Journal, Vol. 10, No. 3, March.

- Bataller, F.J., Rejas, J.G., Bonatti, J., Marchamalo, M. and Martínez, R. (2010). "Detection of hydrothermal alteration using a principal component analysis applied to hyperespectral HyMAP data on the Turrialba volcano, Costa Rica" Geomatica Week International Congress, Barcelona (Spain), 23-25 February 2011.

- Berni, J.A., Zarco-Tejada, P., Suárez, L and Fereres, E. (2010). "Thermal and narrowband multispectral remote sensing for vegetation monitoring from an Unmanned Aerial Vehicle. IEEE Transactions On Geoscience And Remote Sensing, Vol. 47, No. 3., March 2009.

- Borghys, D., Achard, V., Kasen, I. and Perneel, C. (2012). "Comparative evaluation of hyperspec-tral anomaly detection methods in scenes with diverse complexity". In Proc. OPTRO2012 Symposium on Optronics in Defence and Security, Paris, France.

- Cipar, J., Anderson, G. and Cooley, T., (2011). "Active volcano 
monitoring using a space-based short-wave infrared imager". Proceedings WHISPERS 2011, Lisbon (Portugal) 6-9 June.

- Cocks T., R. Jenssen, A. Stewart, I. Wilson, and T. Shields (1998). "The HyMAP Airborne Hyperspectral Sensor: The System, Calibration and Performance" Proc. 1st EARSeL Workshop on Imaging Spectroscopy (M. Schaepman, D. Schläpfer, and K.I. Itten, Eds.), 6-8 October, Zurich, EARSeL, Paris, p. 37- 43.

- Crosta, A. P., Filho, C. R. de Souza, Azevedo, F. and Brodie, C. (2003). "Targeting key alteration minerals in epithermal deposit in Patagonia, Argentina, using ASTER imagery and principal component analysis". International Journal of Remote Sensing, 10, pp. 4233-4240.

- Duran, O. and Petrou, M. (2007). "A time-efficient method for anomaly detection in hyperspectral images". IEEE Transactions on Geoscience and Remote Sensing, Vol. 45, No. 12, December.

- $\quad$ Eismann, M.T., Stocker, A.D. and Nasrabadi, N.M. (2009)."Automated Hyperspectral Cueing for Civilian Search and Rescue" Proceedings of the IEEE, No. 6, June.

- Haboudane, D., Miller, J.R., Tremblay, N., Zarco-Tejada, P.J. and Dextraze, T. (2002). "Integrated narrow-band vegetation indices for prediction of crop chlorophyll content for application to precision agriculture". Remote Sensing of Environment., vol. 81, no. 2/3, pp. 416-426, Aug.

- Hook, S. J. Myers, J. J., Thome, K. J., Fitzgerald, M. and A. B. Kahle (2001). The MODIS, ASTER airborne simulator (MASTER), a new instrument for earth science studies." Remote Sensing of Environment, vol. 76, Issue 1, pp. 93-102.

- Manolakis, D. and Shaw, G. (2002). "Detection Algorithms for Hyperspectral Imaging Aplications". IEEE Signal Processing Magazine, January.

- Malpica, J.A., Rejas, J.G. and Alonso, M.C. (2008). "A projection pursuit algorithm for anomaly detection in hyperspectral imagery". Pattern Recognition 41 (11) pp. 3313-3327, 2008. Nasrabadi, N.M. 
(2008). "Multisensor Joint Fusion and Detection of Mines Using SAR and Hyperspectral". Proceedings of IEEE Sensors 2008 Conference.

- Reed, I.S. and Xiaoli, Y., (1990). "Adaptative multiple-band CFAR detection of an optical pattern with unknown spectral distributio"n. IEEE Transactions on Acoustics. Speech and Signal Processing, Vol. 38, No 10, October.

- Rejas, J.G., Martínez R. and Malpica J.A. (2007). "Hyperspectral remote sensing application for semi-urban areas monitoring". URBAN 2007 4th IEEE GRSS/ISPRS Joint Workshop on Remote Sensing and Data Fusion over Urban Areas, Paris (France) 11-13 April.

- Rejas, J.G., Burillo, F., López, R., Cano, M.A., Sáiz, M.E., and Farjas, M., (2010). "Integrating SAR data and hyperspectral analysis for the archaeological survey of the Segeda city, Spain. 3th International Conference on Remote Sensing in Archaeology, Tiruchirapalli (India)" BAR International Series 2118.

- Rejas, J.G., Martínez-Frías, J., Bonatti, J., Martínez, R. and Marchamalo, M. (2012). "Anomaly detection and comparative analisys of hydrothermal alteration materials trough hyperspectral multisensor data in the Turrialba volcano" XXII ISPRS International Conference, Melbourne, Australia.

- Rondeaux, G., Steven, M. and Baret, F. (1996). "Optimization of soiladjusted vegetation indices" Remote Sening of Environment., vol. 55 , no. 2, pp. 95-107, Feb.

- Rouse, J.W., Haas, R.H., Schell, J.A., Deering, D.W. and Harlan, J.C. (1974). "Monitoring the vernal advancements and retrogradation of natural vegetation". Final Report. NASA/GSFC, Greenbelt, D, p. 371.

- Schaum, A., (2005). "Hyperspectral detection algorithms: Operational, next generation, on the horizon". Proceedings of the 34th Applied Imagery and Pattern Recognition Workshop (AIPR05). 
- Stein, D.W.J., Beaven, S.G., Hoff, L.E., Winter, E.M., Schaum, A.P. and Stocker, A.D. (2002). "Anomaly Detection from Hyperspectral Imagery". IEEE Signal Processing Magazine, January. 